\title{
Flexible triangular formation keeping of marine robotic vehicles using range measurements $^{1}$
}

\author{
Francisco Rego ${ }^{*, * * *}$ Jorge M. Soares ${ }^{*, * * *}$ António Pascoal ${ }^{*, \dagger}$ \\ A. Pedro Aguiar** Colin Jones ${ }^{* * *}$ \\ * Laboratory of Robotics and Systems in Engineering and Science, \\ Instituto Superior Técnico, University of Lisbon, Lisbon, Portugal. \\ ** Department of Electrical and Computer Engineering, Faculty of \\ Engineering, University of Porto, Porto, Portugal. \\ *** Institute of Mechanical Engineering, School of Engineering, École \\ Polytechnique Fédérale de Lausanne, Lausanne, Switzerland. \\ **** Distributed Intelligent Systems and Algorithms Laboratory, School \\ of Architecture, Civil and Environmental Engineering, École \\ Polytechnique Fédérale de Lausanne, Lausanne, Switzerland. \\ $\dagger$ National Institute of Oceanography, Dona Paula, Goa, India.
}

\begin{abstract}
This paper addresses the problem of keeping an autonomous marine vehicle in a moving triangular formation by regulating its position with respect to two leader vehicles. The follower vehicle has no prior knowledge of the path described by the leaders but has access to their heading angle and is able to measure inter-vehicle ranges. It is assumed that the distance between the leaders is constant and known. A control strategy is adopted that generates speed and heading commands so as to drive suitably defined along track and cross track errors to zero. The commands are used as input to local inner loops for yaw and speed control. The paper describes the algorithms derived for range-based control and assesses their performance in simulations using realistic models of the vehicles involved. Tests with three autonomous marine vehicles equipped with acoustic modems and ranging devices allow for the evaluation of the performance of the algorithms in a real-world situation.
\end{abstract}

Keywords: Autonomous Vehicles; Marine Systems; Control Laws; Formation Control; Station Keeping; Triangular Formations.

\section{INTRODUCTION}

Motivated by advances in small embedded processors, sensors, and miniaturized actuators, the development of fleets of autonomous marine vehicles has been gaining momentum worldwide, and shows the potential to drastically improve the means available for ocean exploration and exploitation. It is envisioned that the use of multiple autonomous robotic vehicles acting in cooperation will drastically increase the performance, reliability, and effectiveness of automated systems at sea. Possible scientific and commercial missions include marine habitat mapping, geophysical surveying, and adaptive ocean sampling, to name but a few.

One of the most challenging mission scenarios at sea is underwater habitat mapping in complex 3D environments, where the flexible structure of a fleet of small autonomous underwater vehicles (AUVs) is preferable to a single well-

\footnotetext{
1 This work was supported in part by projects MORPH (EU FP7 under grant agreement No. 288704), CONAV/FCT-PT (PTDC/EEACRO/113820/2009), and the FCT [PEst-OE/EEI/LA0009/2011]. The work of the authors was partially supported by grants SFRH/BD/51929/2012 and SFRH/BD/51073/2010 from Fundação para a Ciência e a Tecnologia.
}

equipped AUV. In this scenario, it is critical that a number of vehicles carrying different sensor suites and navigation equipment maneuver in formation at close range, cooperating towards the acquisition of environmental data. Meeting this objective requires that the vehicles be equipped with advanced systems for networked navigation and control. As an example, we cite a mission in shallow water where one or more surface vehicles (the anchor vehicles) are equipped with advanced sensor suites for absolute georeferencing, such as GPS, so as to follow desired paths or maneuver along arbitrary trajectories in response to episodic events. It is up to the follower vehicles in the fleet to reach and maintain a desired formation with the anchors, effectively moving along at the same speed while acquiring relevant environmental data with complementary sensor suites.

In practice, executing this type of mission without expensive inertial sensor suites requires the follower vehicles to maneuver into formation by relying on measurements of their distances to the leading vehicles and exchanging complementary data. This entails considerable difficulties underwater, as conventional communication and localization systems are unavailable and usually replaced by acoustic devices: acoustic modems that allow the exchange of data, 
and ranging devices that estimate distances by measuring time-of-flight of acoustic signals. These devices exhibit a number of constraints that are inherent to the medium, such as temporary communication losses, outliers in the range measurements, and low bandwidth of the acoustic communication systems. In practice, an important consequence of these limitations is the inability to measure or communicate frequently, with inter-sample times often in the range of seconds, making the problem of underwater range-based multiple vehicle formation keeping very challenging.

Over the past few years, there has been a flurry of theoretical activity in the field of cooperative motion control, as reflected in the large number of publications in networked control systems and robotics. Some of this work falls in the scope of cooperative path following, where a group of vehicles is required to maneuver along prespecified paths while keeping a desired formation pattern (absolute formation control). See Ghabcheloo et al. (2009) for work along these lines with applications in the marine field. In the work reported, each vehicle is required to know its absolute position and those of the neighboring vehicles. This is in contrast with the work in Cao et al. (2007), where the objective is not to do absolute formation control but relative formation control instead. In this situation, each vehicle is only required to know the position of some of the neighbors in its own reference frame. For underwater vehicles, however, this condition is far from trivial.

Representative work in the area of relative formation control includes that of Desai et al. $(1998,2001)$ on the so-called leader-follower formation control problem for a formation graph with an arbitrary number of vehicles. In the work cited, two approaches were proposed using either range-bearing or range-range control, depending on the available sensors. In both approaches, knowledge of the leader motion was assumed. A different strategy is employed in Cao and Morse (2007, 2008), where a solution is proposed for a 4 -vehicle station keeping problem, requiring exclusively range measurements and a decentralized control policy using switched adaptive control. The vehicle dynamics correspond to single integrators in $2 \mathrm{D}$.

In the more recent work of Cao et al. (2011), the authors advance algorithms to coordinate a formation of mobile agents when the agents can only measure the distances to their respective neighbors. This solution requires that subsets of non-neighbor agents cyclically localize the relative positions of their respective neighbor agents while these are held stationary and only then move to reduce the value of a cost function; the latter is nonnegative and assumes the zero value precisely when the inter-vehicle distances in the formation are the prespecifed desired distances. Again, it is assumed that the mobile agents can be described by kinematic points.

Additional related work includes that of Anderson and $\mathrm{Yu}$ (2011), which provides conditions on the range measurements required for each vehicle to infer the relative positions of its neighbors in its own coordinate frame. In the work of Kim et al. (2007), a method is presented for formation keeping of an unmanned aerial vehicle using relative range information. The proposed controller is designed using classical input-out feedback linearization

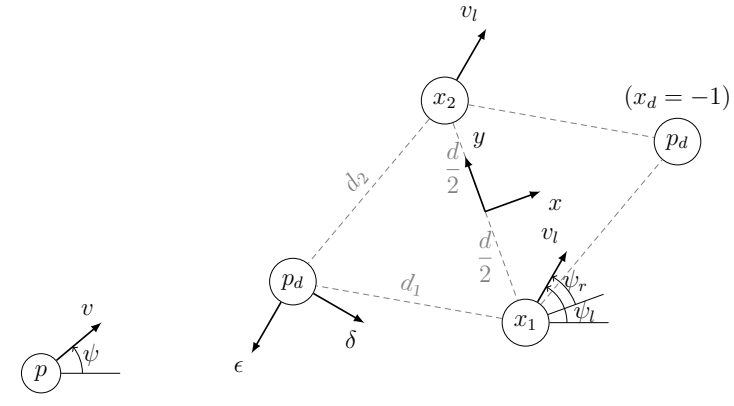

Fig. 1. Formation schematics.

methods. It is important to observe that the performance of any of these methods depends on the accuracy of the range measurements, which are often subject to sensor noise and only available at discrete times. To cope with this limitation, an extended Kalman filter, such as the one proposed in Alcocer et al. (2007), can be useful as part of a range based formation keeping algorithm. A similar approach has been proposed for merging inertial and range information for localization purposes (e.g. Allotta et al. (2011)).

Motivated by the above considerations, Soares et al. (2012, 2013) addressed the simplified problem of maintaining an autonomous vehicle in a moving triangular formation with respect to two leader vehicles that move at the same speed and with constant separation. The follower vehicle has no a priori knowledge of the path described by the leaders and its goal is to follow them by regulating its relative position to a desired point in the formation, using range measurements and the heading of the leaders.

The present paper borrows the framework and control structure proposed in Soares et al. (2012). However, it departs from it in that it deals with a flexible geometry, where the formation is not restricted to the case where the distance to the two leader vehicles is identical and the two-leaders travel side by side. Rather, the leaders follow the same path with one trailing the other. To cope with this new situation, instead of regulating the commonand differential- mode errors defined in the previous work, suitably defined along track and cross track errors are regulated to zero. This set-up is strongly influenced by the mission scenarios adopted in the scope of the EU FP7 project MORPH (Kalwa et al. (2013)), where a formation is spearheaded by a single vehicle equipped with a multibeam echosounder and trailed by a communication coordination vehicle.

We propose a control strategy for the follower vehicle that uses simple feedback laws for speed and heading commands to drive along track and cross track errors to zero. Simulation results using a realistic model of an existing marine vehicle are described and discussed. The performance of the algorithm that we propose is demonstrated in sea trials with the same vehicles, equipped with acoustic modems and ranging devices affected by noise, outliers, and communication losses.

\section{PROBLEM FORMULATION}

The range based formation control problem addressed in this paper can be understood by referring to Fig. 1. The 
objective is to execute a triangular formation keeping maneuver, that is, to drive and maintain a vehicle, henceforth known as the follower, at a desired position with respect to two leader vehicles that run a cooperative path following controller Ghabcheloo et al. (2009). The follower obtains, via an acoustic ranging and communications device, range measurements to each leader, as well as their headings. These measurements have a period of multiple seconds.

The kinematic model for the AUV is written in terms of its speed and heading. In the figure, the follower is at position $p$ with controlled speed $v$ and heading angle $\psi$ measured with respect to an inertial reference frame. The leaders move in cooperation, with equal reference speed and heading denoted $v_{l}$ and $\psi_{l}$, respectively. For simplicity, we assume that the course and heading angles are equal, i.e., there is no current and side-slip is negligible. The two leaders, denoted $x_{1}$ and $x_{2}$, move at a fixed distance $d$ from each other.

To describe the geometry of the formation we define an $x-y$ frame with origin at the midpoint between the leaders. The $y$ axis points from $x_{1}$ to $x_{2}$ and the $x$ axis points $90^{\circ}$ clockwise from the $y$ axis. The desired position of the follower, denoted $p_{d}$, is at a distance $d_{1}$ from $x_{1}$ and $d_{2}$ from $x_{2}$. To disambiguate the two possible locations of $p_{d}$ we introduce a flag $x_{d}$ so that if $x_{d}=1$ then $p_{d}$ is on the negative side of the $x$ axis, and if $x_{d}=-1$ then $p_{d}$ is on the positive side of the $x$ axis.

For controller design purposes we define the along track - cross track, $\epsilon-\delta$ reference frame with origin at $p_{d}$. The along track axis $\epsilon$ points in a direction opposite to that specified by the heading of the leaders, that is, $\psi_{l}+$ $180^{\circ}$. The $\delta$ axis points $90^{\circ}$ anti-clockwise from the $\epsilon$ axis. Written in the $\epsilon-\delta$ frame, the kinematics of the follower vehicle are as follows:

$$
\begin{gathered}
\dot{p_{\epsilon}}=v_{l}-v \cos \left(\psi-\psi_{l}\right) \\
\dot{p_{\delta}}=-v \sin \left(\psi-\psi_{l}\right) .
\end{gathered}
$$

where $p_{\epsilon}$ and $p_{\delta}$ are respectively the $\epsilon$ and $\delta$ coordinates of $p$. The goal is to derive outer-loop feedback laws in $p_{\epsilon}$ and $p_{\delta}$ to drive the follower vehicle to the desired position $p_{d}$, specified by the desired distances $d_{1}$ and $d_{2}$ and by the flag $x_{d}$ (see Fig. 1). If the errors $p_{\epsilon}$ and $p_{\delta}$ go to zero, then $p$ converges to $p_{d}$.

\section{CONTROLLER DESIGN}

The control system, depicted in Fig. 2, consists of multiple discrete modules. The travel time of messages exchanged with the leaders $t^{a c o}$ and the heading of the leaders $\psi_{l}^{a c o}$ are received from the acoustic modem. These signals are then processed to exclude outliers and filtered through Kalman filters yielding estimates of the distance between the follower and $x_{1}\left(\hat{z}_{1}\right)$, the distance between the follower and $x_{2}\left(\hat{z}_{2}\right)$, and the circular mean of the leaders' angle $\left(\hat{\psi}_{l}\right)$. The distance estimates $\hat{z}_{1}$ and $\hat{z}_{2}$ are used to compute $\left(p_{\epsilon}, p_{\delta}\right)$, the estimated position of the follower in the $\epsilon-\delta$ frame. Using the $\epsilon$ coordinate, a velocity controller computes a command of desired speed $u_{d}$ which is then linearly transformed to a common mode command. Finally, with the leaders' heading estimate $\hat{\psi}_{l}$ and the $\delta$ coordinate, a heading controller yields a reference for the follower's

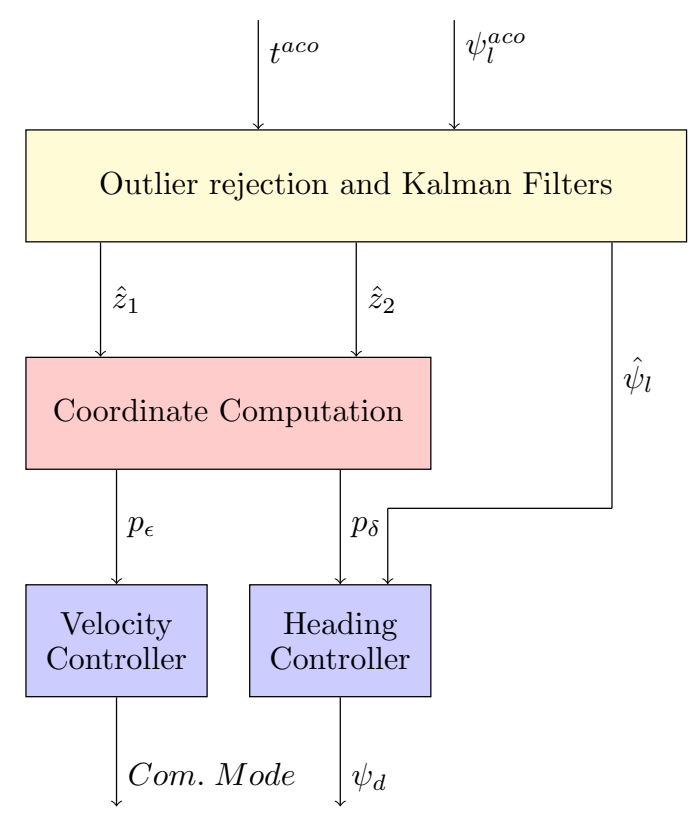

Fig. 2. Control system diagram.

heading angle $\psi_{d}$. The details of each module are presented in the subsections below.

\subsection{Outer-loop feedback}

One distinctive characteristic of this work is the use of simple control laws that separately regulate the desired linear velocity $u_{d}$ and heading $\psi_{d}$. These are then fed to inner loop controllers specific to the vehicle.

The control law for the desired velocity is given by:

$$
u_{d}=\operatorname{sat}\left(k_{u p} p_{\epsilon}+k_{u i} \int_{0}^{t} p_{\epsilon} d \tau+v_{l_{\text {nom }}}\right),
$$

where $k_{u p}$ is the proportional gain, $k_{u i}$ is the integral gain and $v_{l_{\text {nom }}}$ is a scenario-configurable nominal velocity. While not strictly required, the use of a $v_{l_{n o m}}$ close to the leader speed accelerates the convergence to the desired speed and position. The final value is run through a saturation function that limits the output to $\left[v_{\min }, v_{\max }\right]$. An integration clamping anti-windup scheme is adopted, i.e. the integration is interrupted when the control variable saturates and the control error and control variable have the same sign.

The control law for $\psi_{d}$ is given by:

$$
\psi_{d}=\hat{\psi}_{l}+\operatorname{sat}\left(k_{\psi p} p_{\delta}+k_{\psi i} \int_{0}^{t} p_{\delta} d \tau\right) .
$$

The heading controller tracks the reference heading estimated from the information sent by the leaders, and adds a PI controller on the error $p_{\delta}$. The output of the PI controllers is saturated to $[-0.5 \mathrm{rad}, 0.5 \mathrm{rad}]$ so that, even for large errors, the vehicle does not move in a direction opposite the leaders'. As in the case of the velocity control law, an integration clamping anti-windup scheme is implemented.

With those control laws, defining $\eta:=v_{l}-k_{u i} \int_{0}^{t} p_{\epsilon} d \tau-$ $v_{l_{\text {nom }}}$ and $\xi:=-k_{\psi i} \int_{0}^{t} p_{\delta} d \tau$ and neglecting the inner loop dynamics, i.e. considering $v=u_{d}$ and $\psi=\psi_{d}$ we may linearize the kinematics of the follower about $p_{d}$ yielding 


$$
\left[\begin{array}{c}
\dot{p_{\epsilon}} \\
\dot{\eta}
\end{array}\right]=\left[\begin{array}{cc}
-k_{u p} & 1 \\
-k_{u i} & 0
\end{array}\right]\left[\begin{array}{c}
p_{\epsilon} \\
\eta
\end{array}\right]
$$

and

$$
\left[\begin{array}{c}
p_{\delta} \\
\dot{\xi}
\end{array}\right]=\left[\begin{array}{cc}
-v_{l} k_{\psi p} & v_{l} \\
-k_{\psi i} & 0
\end{array}\right]\left[\begin{array}{c}
p_{\delta} \\
\xi
\end{array}\right] \text {. }
$$

The characteristic polynomial for the $p_{\epsilon}$ dynamics is $s^{2}+$ $s k_{u p}+k_{u i}$ and the characteristic polynomial for the $p_{\delta}$ dynamics is $s^{2}+s v_{l} k_{\psi p}+v_{l} k_{\psi i}$. Therefore we may select the gains for $p_{\delta}$ as $k_{u p}:=2 \zeta_{u} \omega_{n u}$ and $k_{u i}:=\omega_{n u}^{2}$ and the gains for $p_{\epsilon}$ as $k_{\psi p}:=\zeta_{\psi} \omega_{n \psi} / v_{l}$ and $k_{\psi i}:=\omega_{n \psi}^{2} / v_{l}$.

\subsection{Outlier rejection}

Due to the nature of acoustic ranging, erroneous readings are frequent, especially in shallow waters with irregular seabed topography. Therefore, the range samples received must be filtered for outliers. A measurement $m$ is accepted if it is inside the interval

$$
\left[a-s_{\max } \cdot\left(t-t_{l}\right), a+s_{\max } \cdot\left(t-t_{l}\right)\right]
$$

For $z:=v_{\text {sound }} \cdot t^{\text {aco }}$ to be accepted, we consider $s_{\max }=$ $v_{\max }$, the maximum speed of the vehicle, and for $\psi_{l}^{a c o}$ to be accepted we consider $s_{\max }=\omega_{\max }$, the preset maximum angular speed.

When a measurement is accepted, $a$ and $t_{l}$ are updated according to $t_{l}=t$ and $a(k+1)=\left(1-k_{a c c}\right) \cdot a(k)+k_{a c c} \cdot m$, where $k_{a c c}=0.5$. When a measurement is not accepted but is inside the interval

$$
\left[a-4 s_{\max } \cdot\left(t-t_{l}\right), a+4 s_{\max } \cdot\left(t-t_{l}\right)\right]
$$

then $a$ is updated as $a(k+1)=\left(1-k_{r e j}\right) \cdot a(k)+k_{r e j} \cdot m$, where $k_{r e j}=0.25$. A measurement outside these ranges is discarded.

\subsection{Kalman filter}

The long period between samples and relatively fast error dynamics require the use of an estimator to improve the behavior of the controllers. The problem is further exacerbated in the presence of packet loss, frequent for some scenarios and particular modem alignment conditions. We chose to use a Kalman filter, with a model as follows:

$$
x(k+1)=A x(k)+w
$$

where $A$ is defined as

$$
A=\left[\begin{array}{ll}
1 & 1 \\
0 & 1
\end{array}\right]
$$

and $w$ is process noise with covariance matrix $Q$. The output equation is

$$
y(k)=H x(k)+v
$$

where $H$ is defined as

$$
H=\left[\begin{array}{ll}
1 & 0
\end{array}\right]
$$

and $v$ is process noise with covariance matrix $R$. The sampling period considered is 0.2 seconds. Notice that we only perform the update step when a measurement is received, i.e. during most samples only the prediction step is performed.

The estimated states are $\hat{z}_{1}, \hat{z}_{2}$ and $\hat{\psi}_{l}$ with one Kalman filter for each state. The Kalman filters are updated each time a new measurement is accepted. The estimated ranges $\hat{z}_{i}$ are updated with $y=v_{\text {sound }} t_{i}^{a c o}$ and the estimated leaders' average heading $\hat{\psi}_{l}$ is updated with the circular mean of the last accepted headings from each leader.

\section{SIMULATION RESULTS}

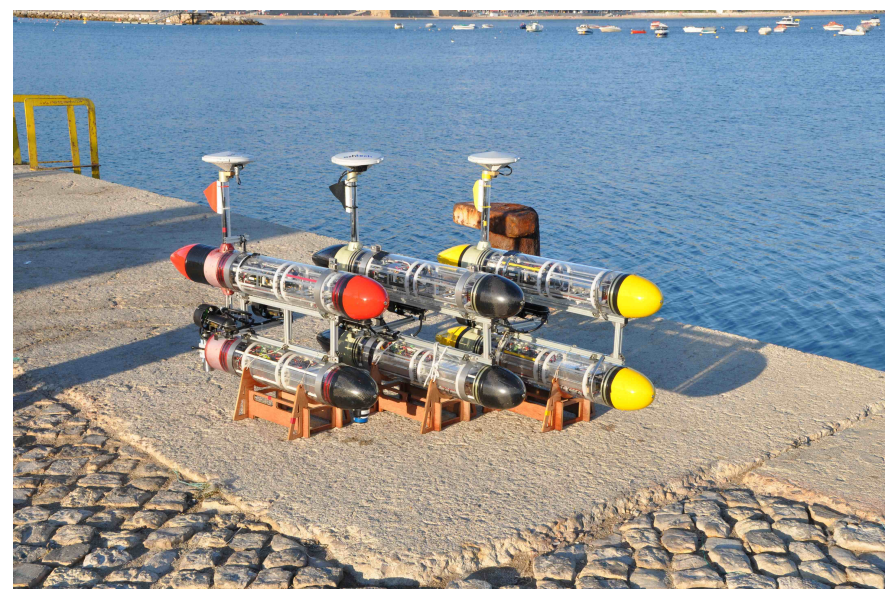

Fig. 3. Medusa vehicles.

Simulations were carried out in order to evaluate the performance of the algorithm in preparation for the sea trials. For this purpose, we used a Simulink model of the MEDUSA AMV shown in Fig. 3, with the inner loop controller for heading described in Ribeiro et al. (2012).

The two leaders follow the same path at $0.5 \mathrm{~m} / \mathrm{s}$ with the follower at starboard of the leaders. Each vehicle is $15 \mathrm{~m}$ away from the others, that is, $\psi_{l}=-\pi / 2, x_{p}=1$ and $d_{1}=d_{2}=d=15 \mathrm{~m}$. The acoustic modems of each of the leader vehicles transmit information with a period of $4 s$. The simulation time step is of $0.2 s$. Sensing and ranging imperfection are taken into account, with a packet loss rate of $40 \%$ and added Gaussian ranging noise with $\sigma=0.45 \mathrm{~m}$. The parameters of the ROF controller are the following:

$$
\begin{aligned}
& \text { - } \omega_{n u}-0.041 \\
& \text { - } \zeta_{u}-0.82 \\
& \text { - } \omega_{n \psi}-0.0022 \\
& \text { - } \zeta_{\psi}-8.9
\end{aligned}
$$

The simulated paths of the vehicles can be seen in Fig. 4 and the along track and cross track errors are shown in Fig. 5.

From Figures 4 and 5 we can conclude that the control algorithm achieves the objective of keeping the vehicles in formation, and is able to cope with packed loss and sensor noise.

After an initial transient phase, the along track and cross track errors are bounded between $-5 \mathrm{~m}$ and $5 \mathrm{~m}$. There is, nevertheless, room for improvement on the range measurement filters, especially to mitigate the effect of packet losses. A possible enhancement would be using an extended Kalman filter (EKF) that takes into account the input commands.

\section{SEA TRIALS}

To verify the performance of the ROF controller, a trial was performed at Parque das Nações, Lisbon, Portugal, 


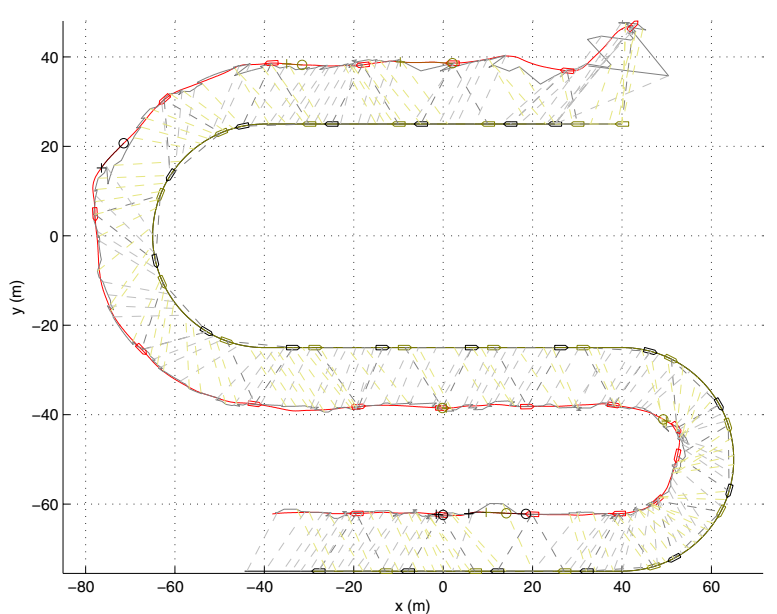

Fig. 4. Simulated vehicle paths. The solid red line is the actual path followed by the vehicle, the gray solid line represents the computed path with range measurements, and the dashed lines represent range measurements. The segments between a circle and a cross represent periods during which no range measurement was received from one of the vehicles in the preceding 12 seconds.
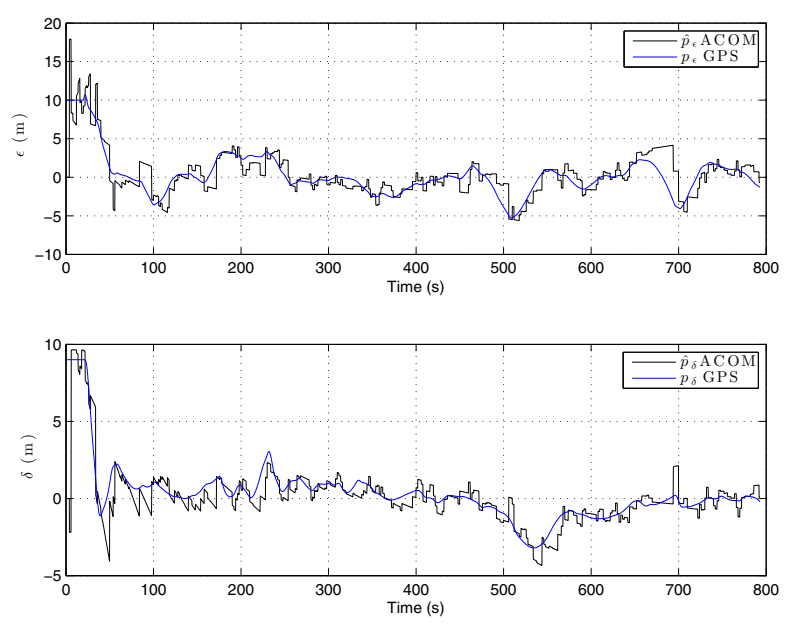

Fig. 5. Along track $\left(p_{\epsilon}\right)$ and cross track $\left(p_{\delta}\right)$ errors during simulation. The black line represents the estimated errors from the ranges measured by the virtual acoustic modems. The blue line indicates the exact along track and cross track errors, as if they were computed using continuous exact measurements.

in a closed harbor with shallow waters and no boat traffic. The MEDUSA AMVS, developed at the LARSyS/ ISR/IST, Lisbon, Portugal, were used in the sea trials.

The vehicles implement a Dynamic Long Baseline method conceived by the NATO Centre for Maritime Research and Experimentation (CMRE) atop functionalities present in the EvoLogics modems (Kebkal et al. (2012)). Each node has the ability to accurately timestamp, in a local clock, the incoming and outgoing packets. Every packet sent is then used as a query and yields N-1 replies in a distributed LBL scheme, considering the time differences between queries receptions and reply transmission. Since the nodes are at close distance, rigid time-division multiple access (TDMA) is used without incurring a large penalty

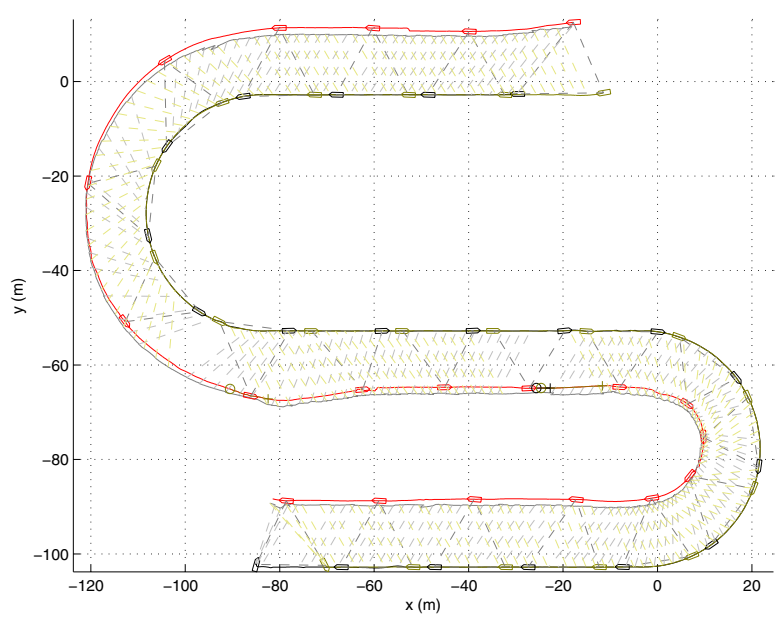

Fig. 6. Vehicle paths during trials. The follower vehicle is plotted in red and the leaders are plotted in black and yellow.

in channel capacity. Heading exchanges are piggybacked on these localisation packets. At the end of each round, and in the absence of losses, all vehicles will know the distance to and heading of every other vehicle in communication range. This is an improvement over the simple ping-reply model used in Soares et al. (2013).

During this trial, we again considered the mission described in Section 4. The paths described by the vehicles during the trial are shown in Fig. 6 and the along track and cross track errors are shown in Fig. 7. Aside from minimal disturbances, the follower is able to better track the leaders than observed in the simulation. This suggests that either our vehicle model is not a sufficiently accurate representation of the real vehicles and/or that our simulation overestimates sensor noise and packet losses. The latter is found to be true, with packet loss during trials recorded as $8.12 \%$, compared to the $40 \%$ rate used in simulation.

\section{CONCLUSION}

This paper proposed a solution to a three-vehicle formation keeping problem where a follower moves in a triangular formation with two leader vehicles. The follower has no knowledge of the path taken by the leaders, and uses only inter-vehicle range measurements, the predefined relative position between the two leaders and their headings.

Preliminary simulation results were described for a lawnmower motion using a dynamic model of the Medusa vehicles developed at ISR/IST. The results show good performance with error bounded to a $5 m$ window.

We have also addressed the implementation and testing of the algorithm in marine scenarios using real marine vehicles. The algorithm is able to deal with range measurements that are only available at discrete points in time, with a period of several seconds. Furthermore, these measurements are affected by sensor noise and outliers, as well as communication delays and temporary losses. 

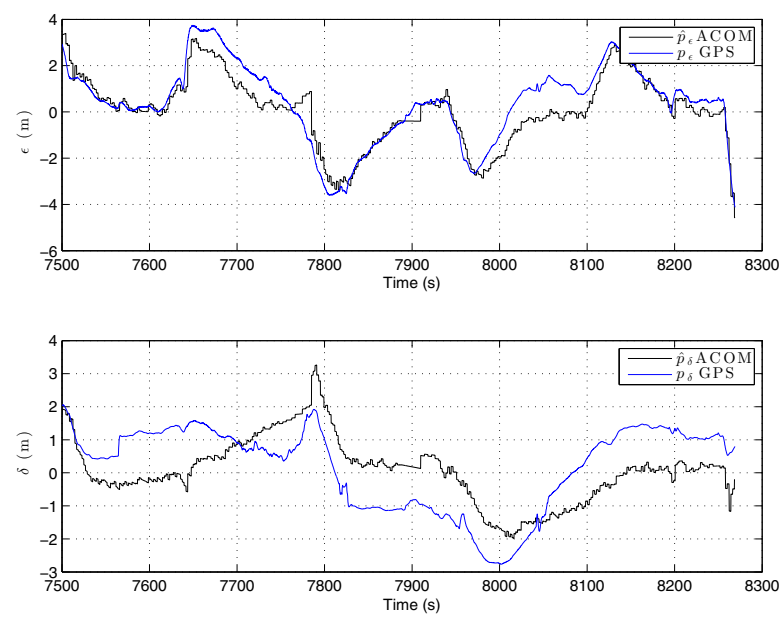

Fig. 7. Along track $\left(p_{\epsilon}\right)$ and cross track $\left(p_{\delta}\right)$ errors during trials. The black line represents the estimated errors from the ranges measured by the acoustic modems. The blue line indicates the along track and cross track errors computed using RTK GPS measurements.

Future steps will include the analysis of the performance and robustness of the algorithm proposed. A simple but significant improvement would be to have the estimators take into account the commands given to the vehicle, as well as other data, namely from inertial sensors.

\section{ACKNOWLEDGEMENTS}

We thank M. Gallieri for his early work on this subject, and the DSOR team for their efforts in the development of the MEDUSA vehicles and their support and collaboration on the planning and execution of our sea trials. We also thank the NATO CMRE centre for the development of the ranging algorithm and their generous support and expertise during sea trials.

\section{REFERENCES}

Alcocer, A., Oliveira, P., and Pascoal, A. (2007). Study and implementation of an EKF GIB-based underwater positioning system. Control engineering practice, 15(6), 689-701.

Allotta, B., Pugi, L., Costanzi, R., and Vettori, G. (2011). Localization algorithm for a fleet of three AUVs by INS, DVL and range measurements. In Advanced Robotics (ICAR), 2011 15th International Conference on, 631636. doi:10.1109/ICAR.2011.6088608.

Anderson, B.D. and Yu, C. (2011). Range-only sensing for formation shape control and easy sensor network localization. In Control and Decision Conference $(C C D C)$, 2011 Chinese, 3310-3315. IEEE.

Cao, M. and Morse, A. (2007). Station keeping in the plane with range-only measurements. In American Control Conference, 200\%. ACC'0\%, 5419-5424. IEEE.

Cao, M., Morse, A., Yu, C., Anderson, B., and Dasgupta, S. (2007). Controlling a triangular formation of mobile autonomous agents. In Decision and Control, 200746 th IEEE Conference on, 3603-3608. IEEE.

Cao, M. and Morse, A.S. (2008). The use of dwell-time switching to maintain a formation with only range sensing. In Communications, Control and Signal Processing,
2008. ISCCSP 2008. 3rd International Symposium on, 954-959. IEEE.

Cao, M., Yu, C., and Anderson, B. (2011). Formation control using range-only measurements. Automatica, 47(4), 776-781.

Desai, J.P., Ostrowski, J.P., and Kumar, V. (2001). Modeling and control of formations of nonholonomic mobile robots. Robotics and Automation, IEEE Transactions on, 17(6), 905-908.

Desai, J.P., Ostrowski, J., and Kumar, V. (1998). Controlling formations of multiple mobile robots. In Robotics and Automation, 1998. Proceedings. 1998 IEEE International Conference on, volume 4, 2864-2869. IEEE.

Ghabcheloo, R., Aguiar, A.P., Pascoal, A., Silvestre, C., Kaminer, I., and Hespanha, J. (2009). Coordinated path-following in the presence of communication losses and time delays. SIAM Journal on Control and Optimization, 48(1), 234-265.

Kalwa, J., Pascoal, A., Ridao, P., Birk, A., Eichhorn, M., Brignone, L., Caccia, M., Alves, J., and Santos, R. (2013). The european R\&D-project morph: Marine robotic systems of self-organizing, logically linked physical nodes. In Proc. MCMC 2012. URL http: //morph-project.eu.

Kebkal, O., Kebkal, K., and Bannasch, R. (2012). Longbaseline hydro-acoustic positioning using d-mac communication protocol. In OCEANS, 2012 - Yeosu, 1-7. doi: 10.1109/OCEANS-Yeosu.2012.6263529.

Kim, S., Ryoo, C.K., Choi, K., and Park, C. (2007). Multi-vehicle formation using range-only measurement. In Control, Automation and Systems, 2007. ICCAS'O\%. International Conference on, 2104-2109. IEEE.

Ribeiro, J., Aguiar, A.P., and Pascoal, A.M. (2012). Motion control design for the medusa robotic vehicle with experimental results. Technical report, LARSyS.

Soares, J.M., Aguiar, A.P., Pascoal, A.M., and Gallieri, M. (2012). Triangular formation control using range measurements: An application to marine robotic vehicles. In Proceedings of the IFAC Workshop on Navigation, Guidance and Control of Underwater Vehicles, Porto, Portugal.

Soares, J.M., Aguiar, A.P., Pascoal, A.M., and Martinoli, A. (2013). Joint ASV/AUV range-based formation control: Theory and experimental results. In Proceedings of the IEEE International Conference on Robotics and Automation, Karlsruhe. 